\title{
The effects of binaries on the evolution of Wolf-Rayet type spectral features in starbursts
}

\author{
J. Van Bever and D. Vanbeveren
}

\author{
Astrophysical Institute, Vrije Universiteit Brussel, Pleinlaan 2, 1050 Brussels, Belgium \\ e-mail: dvbevere@vub.ac.be
}

Received 18 March 2002 / Accepted 27 November 2002

\begin{abstract}
In the present paper we investigate in detail the effects of binaries with initial period between 1 day and 10 years on theoretical simulations of Wolf-Rayet (WR) type spectral features in starbursts. We focus on the evolution of the nebular $H_{\beta}$ line in starburst in general, on the intensity ratios $I$ (nebular He II $\lambda 4686) / I\left(H_{\beta}\right), I$ (blue bump) $/ I\left(H_{\beta}\right)$, and $I($ red bump $) / I\left(H_{\beta}\right)$ as a function of the equivalent width of $H_{\beta}$ of WR galaxies in particular. The binary evolutionary processes that dominate the evolution of the considered spectral features are the Roche lobe overflow in case Br systems, the mass transfer efficiency, and the merger rate. We show that the predictions on nebular He II depend critically on the uncertainties in the theory of WR atmospheres and particularly on uncertainties in the treatment of the subsonic velocity region of the WR wind. The observations of low metallicity starbursts are best reproduced by a theoretical model with a significant number of binaries and with a metallicity-dependent WR wind.
\end{abstract}

Key words. stars: evolution - stars: binaries: general - stars: Wolf-Rayet

\section{Introduction}

Starburst galaxies are the seats of rapid ongoing star formation. It has become clear that they are key astrophysical objects which play an essential role in the evolution of galaxies in particular, and the cosmos in general. Theoretical modelling of massive starbursts until now has focussed mainly on the effects of single star evolution and binaries were ignored (Leitherer et al. 1999; Conti 2000). There is however an overwhelming evidence that a large fraction of all massive stars have a close companion (see reviews by Mermilliod 2001; Van Rensbergen 2001; Mason et al. 2001, and references therein) and a discussion of the effects of binaries on the content of stellar populations is necessary to make starburst studies more reliable.

To study these effects it is essential to have a population model that includes all known aspects of single and binary evolution in combination with detailed stellar evolutionary calculations. The Brussels population NUMBER synthesis (PNS) model and our extended stellar evolutionary models have been described by Vanbeveren et al. (1998a, b, c) and by Van Bever $\&$ Vanbeveren (1998), and are summarized in Sect. 2 (see also Vanbeveren 2001).

Van Bever et al. (1999) investigated the consequences of binaries on photoionization models, in particular on the evolution of the nebular Balmer $\alpha\left(H_{\alpha}\right)$ and Balmer $\beta\left(H_{\beta}\right)$ lines. The expected hard X-ray emission $(E \geq 2 \mathrm{keV})$ was

Send offprint requests to: J. Van Bever, e-mail: jvbever@vub.ac.be considered by Van Bever \& Vanbeveren (2000). Schaerer \& Vacca (1998) and Dionne (1999) presented models for WR and O star populations in young starbursts and added a discussion on possible effects of binaries. However, in both papers, the authors adopted a binary evolutionary model which is far too simple and many binary evolutionary aspects were ignored. Here we will investigate in detail the influence of binaries on the evolution of a collection of WR emission lines grouped into the so called blue WR bump (i.e. N III/N V $\lambda 4640$, C III/C IV $\lambda 4650$ and He II $\lambda 4686$ and its nebular contribution), and in the so called red WR bump (i.e. the carbon multiplet C IV $\lambda \lambda 5808$ 5812). In Sect. 3, we describe our population spectral synthesis code. Observations of starbursts which are important for the present paper are discussed in Sect. 4, whereas Sect. 5 deals with the results.

\section{The Population Number Synthesis (PNS) model}

A PNS code with single stars and interacting binaries has to account for:

- the evolution of single stars;

- the evolution of case A, case $\mathrm{Br}$, case $\mathrm{Bc}$ and case $\mathrm{C}$ binaries with mass ratio $q>0.2$ ( $q$ is the mass of the secondary divided by the mass of the primary; the primary is always defined as the star which is originally the most massive component), accounting in detail for the effects of Roche lobe overflow (RLOF), mass transfer, mass loss from the system and common envelope evolution; 
- the evolution of binaries with small mass ratio $(q \leq 0.2)$ which is governed by the spiral-in process;

- the evolution of mergers;

- the effects of the (asymmetric) supernova ( $\mathrm{SN}$ ) explosion on binary parameters;

- a detailed treatment of the evolution of the binary periods, which depends critically on the physics of the previously listed processes.

A PNS code follows the evolution of a population of single stars and binaries by adopting the following stellar distribution functions:

- the initial mass distribution IMF: we use a Salpeter type distribution (Salpeter 1955) for the single star and binary primary initial mass functions. Notice that the conclusions of the present paper do not critically depend on the adopted IMF;

- the binary mass ratio distribution $\phi(q)$ : we made our simulations for a distribution where the number of systems increases as $q$ decreases (Hogeveen 1991, 1992; we will further use the term "Hogeveen distribution"), a flat distribution, and a function that peaks at unity (Garmany et al. 1980);

- the initial binary period distribution is flat in the $\log P$ (Popova et al. 1982; Vereshchagin et al. 1987, 1988);

- asymmetric supernova explosions induce neutron star kicks, which drastically affect the binary periods. We assume that the kicks are randomly directed in space whereas their magnitudes are $\chi^{2}$-distributed, in correspondence with the observational study of Lorimer et al. (1997). The adopted average value is expressed as $\left\langle v_{\text {kick }}\right\rangle$. We explore the consequences when different values are used.

Our PNS code was described in the papers cited in the introduction. It relies on a large set of stellar evolutionary computations of intermediate mass and massive single stars and binary components, with a moderate amount of convective core overshooting and for different initial chemical compositions $(0.001 \leq Z \leq 0.02)$. The calculations of the massive star evolution were performed accounting for recent stellar wind mass loss rate $(\dot{M})$ formalisms (see also Sect. 2.1).

We have over 1000 evolutionary calculations of massive close binaries in which the evolution of the mass gainer is followed up to the end of its core helium burning $(\mathrm{CHeB})$ phase. One of the crucial parameters here is the amount of matter lost by the mass loser due to RLOF that is (or can be) transferred during the RLOF to the mass gainer, i.e. the parameter $\beta$ as it was originally introduced by Vanbeveren et al. (1979). We add a short discussion on the value of $\beta$ in Sect. 2.2.

Mergers are defined as binaries whose components coalesce at some stage during the binary evolution. We will show that mergers may play an important role in population spectral synthesis. In Sect. 2.3 we will discuss our adopted merger model and we will outline in how far the predicted number of mergers depends on $\beta$.

\subsection{Stellar wind mass loss rate formalisms in massive stars}

The evolution of a massive star is critically affected by three stellar wind mass loss phases: the OB phase including the possibility for the object to become a luminous blue variable (LBV), the red supergiant (RSG) phase and the WR phase.

\subsubsection{Stars with initial mass $\geq 40 M_{\odot}$ : The "LBV scenario"}

Based on the observations of LBVs one may suspect that stars with initial mass $\geq 40 M_{\odot}$ experience an LBV phase at the end of core hydrogen burning and/or hydrogen shell burning, which is accompanied by a very violent stellar wind mass loss phase (Humphreys \& Davidson 1994). The lack of RSGs with initial mass $\geq 40 M_{\odot}$ (corresponding roughly to stars with $M_{\text {bol }} \leq-9.5$, Humphreys \& McElroy 1984) may be attributed to this process so that a working hypothesis for stellar evolutionary calculations may be the following:

the mass loss rate during the $L B V+R S G$ phase of a star with initial mass $>40 M_{\odot}$ must be sufficiently large to assure a RSG phase which is short enough to explain the lack of observed RSGs with $M_{\text {bol }} \leq-9.5$.

Obviously, also binary components with an initial mass $\geq 40 M_{\odot}$ will obey this criterium which means that the following scenario applies: when a primary with initial mass $\geq 40 M_{\odot}$ starts its $L B V$ phase before the RLOF (case B and case $C$ systems), the evolution is governed by the LBV stellar wind, and the RLOF (and thus mass transfer) is suppressed. The RLOF in case A systems in this mass range may not be avoided but in our PNS code we treat them in the same way as Case B. The consequences of this simplification will be discussed (Sect. 5.2).

Also the LMC and the SMC show a deficiency of RSGs with $M_{\text {bol }} \leq-9.5$ (Humphreys \& McElroy 1984) so that the single star and binary evolutionary scenario outlined above may apply in low metallicity regions as well. Notice that this "LBV scenario" was introduced more than a decade ago (Vanbeveren 1991), and we used it in all our PNS calculations since 1997.

\subsubsection{RSG mass loss}

Since 1997, we apply a RSG stellar wind mass loss formalism which is based on observations of stars in the Magellanic Clouds by Jura (1987) and Reid et al. (1990). More details are given in the papers in which we describe our PNS code (see the introduction). Notice that we assume that the RSG stellar wind mass loss rate depends on the metallicity $(\propto \sqrt{Z})$. As a consequence our evolutionary calculations of single stars with initial mass $20 M_{\odot} \leq M<40 M_{\odot}$ are distinctly different from those of the Geneva team. This obviously means that the population of single WR stars predicted by our PNS models is different from the population of WR single stars predicted by those of Leitherer et al. (1999) which uses the Geneva evolutionary tracks (see also Sect. 5.1). 
Table 1. The luminosity and the mass loss rates of WR stars determined with NLTE atmosphere models and assuming nonhomogeneous stellar winds. We compare with values predicted by Eq. (1) and by the formula proposed by Nugis \& Lamers (2000, NL).

\begin{tabular}{lllll}
\hline \hline WR number & $\log L$ & $\log \dot{M}$ & Eq. (1) & NL \\
\hline WR6 $^{\mathrm{a}}$ & 5.45 & -4.4 & -4.5 & -4.8 \\
WR147 $^{\mathrm{b}}$ & 5.65 & -4.6 & -4.3 & -4.4 \\
WR111 $^{\mathrm{c}}$ & 5.3 & -4.8 & -4.7 & -4.9 \\
WR90 $^{\mathrm{d}}$ & 5.5 & -4.6 & -4.5 & -4.7 \\
WR135 $^{\mathrm{d}}$ & 5.2 & -4.9 & -4.8 & -4.8 \\
WR146 $^{\mathrm{d}}$ & 5.7 & -4.5 & -4.3 & -4.4 \\
WR11 $^{\mathrm{c}}$ & 5.0 & -5.1 & -5.0 & -5.0 \\
WR123 $^{\mathrm{f}}$ & 5.7 & -4.14 & -4.3 & -4.5 \\
WR139 $^{\mathrm{g}}$ & 5 & -5 & -5 & -4.5 \\
OB10-WR1 $^{\mathrm{h}}$ & 5.7 & -4.3 & -4.3 & -4.5 \\
\hline
\end{tabular}

a Schmutz (1997).

b Morris et al. (2000).

c Hillier \& Miller (1999).

d Dessart et al. (2000).

e de Marco et al. (2000).

f Nugis et al. (1998).

$g$ Underhill et al. (1990).

h Smartt et al. (2001).

\subsubsection{WR mass loss}

Using a hydrodynamic atmosphere code in which the stellar wind is assumed to be homogeneous, Hamann et al. (1995) determined $\dot{M}$ values for a large number of WR stars. Since then evidence has grown that these winds consist of clumps and that an homogeneous model overestimates $\dot{M}$, typically by a factor 2-4 (Hillier 1996; Moffat 1996; Schmutz 1996; Hamann \& Koesterke 1998). Vanbeveren et al. (1998a, b, c) published massive single star and binary evolutionary calculations in which the effects of the lower WR mass loss rates, as inferred from the wind papers listed above, were studied. We assumed a simple relation between $\dot{M}$ (in $M_{\odot} / \mathrm{yr}$ ) and the stellar luminosity $L$ (in $L_{\odot}$ ), $\log \dot{M}=a \log L+b$, and we tried to find appropriate values of the constants $a$ and $b$ accounting for the following criteria and observations known at that time:

- the WN5 star HD 50896 (WR 6) has a luminosity $\log L=$ 5.6-5.7 and a $\log \dot{M}=-4.4 \pm 0.15$ (Schmutz 1996; Schmutz et al. 1996);

- the $\log \dot{M}$ of the WNE component of the binary V444 Cyg (WR 139) resulting from the observed orbital period variation is approximately -5 (Khaliullin et al. 1984; Underhill et al. 1990). Its orbital mass is $9 M_{\odot}$ and, using a massluminosity relation holding for WNE binary components (Vanbeveren \& Packet 1979; Langer 1989) it follows that its $\log L$ equals 5 ;

- the observations of the masses of black hole components in $\mathrm{X}$-ray binaries indicate that stars with initial mass $>40 M_{\odot}$ should end their life with a mass larger than $10 M_{\odot}$ (i.e. the mass of the star at the end of $\mathrm{CHeB}$ );

- the WN/WC number ratio predicted by a PNS code depends on the WR mass loss formalism used in the stellar

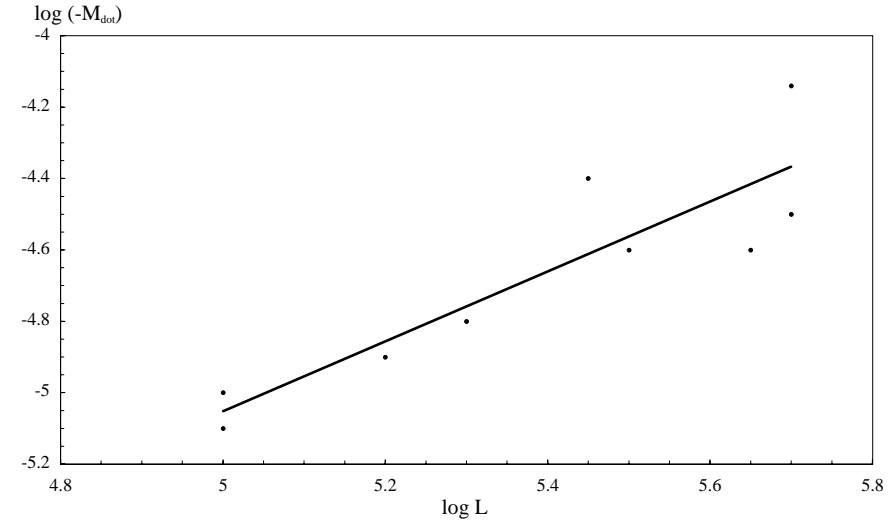

Fig. 1. Observed mass loss rates versus luminosity of WR stars. The line corresponds to Eq. (1) and the filled circles represent the atmospheric model results of Table 1.

evolutionary calculations. Therefore, we looked for $a$ and $b$ values which predict the observed WN/WC number ratio $(\approx 1)$ for the solar neighbourhood.

This exercise allowed us to propose the following relation:

$\log \dot{M}=\log L-10$.

Since then, more WR stars have been investigated with detailed atmosphere codes, some of which included the effects of the clumping. They are listed in Table 1. Figure 1 illustrates that Eq. (1) still fits these new observations fairly well. Nugis \& Lamers (2000, NL) proposed an alternative WR mass loss relation. Table 1 compares predictions from our Eq. (1) and from the NL formula with the spectroscopic results of the WR stars which were investigated with the detailed atmosphere codes. It can be concluded that both formalisms imply similar accuracies. The $Z$-dependency of WR mass loss rates deserves some attention. Nugis \& Lamers adopt a $\dot{M}$ relation which is $Z$-dependent where, as usual, $Z$ is defined as the abundance of all elements heavier than helium. This means that they intrinsically assume that WN and WC stars with the same luminosity and belonging to the same stellar environment (thus having a similar heavy metal abundance) may have quite a different mass loss rate. However, there is no observational evidence that this is the case. It is generally accepted that the WR type stellar wind is radiation driven and therefore one may expect that the heavy metals are the main drivers. On that line of argument it is more plausible that the mass loss rate depends roughly on some power $\zeta$ of the heavy metal abundance of the WR star (which is roughly proportional to the heavy metal abundance of the WR progenitor population). Hamann \& Koesterke (2000) investigated $18 \mathrm{WN}$ stars in the LMC. When these data are plotted in a $\dot{M}-L$ diagram, there seems to be no obvious relation. However, as an exercise, Vanbeveren (2001) used a linear relation similar to Eq. (1) and shifted the line until the sum of the distances between observed values and predicted ones was smallest (a linear regression where the slope of the line is fixed). This resulted in a shift of -0.2 which, accounting for the heavy metal abundance of the Solar neighbourhood and of the LMC, corresponds to $\zeta=0.5$. To be more conclusive, many more observations are needed where detailed analysis may give reliable 
stellar parameters, but from this exercise it looks as if the WR mass loss rates depend on the heavy metal abundance. We will explore the consequences when

- the mass loss rate is independent from the chemical abundance of the envelope of the hydrogen deficient $\mathrm{CHeB}$ star (i.e. we always use Eq. (1));

- the mass loss rate depends on the primordial metallicity $Z$ of the stellar population, i.e.

$$
\log \dot{M}=\log L-10+\log \sqrt{\frac{Z}{Z_{\odot}}} .
$$

We will illustrate in Sect. 5 to what extent population spectral synthesis simulations of WR lines depend on the adopted WR mass loss rate formalism. Appendix A points out how critical the effect of the WR mass loss rate is on the final stellar masses prior to core collapse, as well as on the WR lifetimes (and thus on WR population synthesis).

In 1997-1998, we calculated evolutionary tracks of massive single stars and binary components adopting Eq. (1) to compute the stellar wind mass loss rate at the moment the star becomes a hydrogen deficient $\mathrm{CHeB}$ object. These tracks are part of all the evolutionary models used by the PNS code. All our PNS results published since 1997-1998 (see the papers cited in the introduction) rely on these models, thus rely on Eq. (1). Accounting for the discussion above we will use the same evolutionary models for the present paper.

\subsection{The RLOF in massive binaries: conservative or not?}

It is likely that case $\mathrm{B} \backslash \mathrm{C}$ binaries with initial primary mass $\geq 40 M_{\odot}$ avoid RLOF due to a LBV type stellar wind mass loss phase which precedes the RLOF (see also Sect. 2.1.1). At the beginning of the RLOF in a case $\mathrm{Bc}$ or case $\mathrm{C}$ binary with initial primary mass $<40 M_{\odot}$, the system enters a common envelope phase (Webbink 1984), and it is likely that all mass lost by the loser leaves the system $(\beta=0)$. A parameter which can make a significant difference between population spectral synthesis with or without binaries is the value of $\beta$ in case $\mathrm{Br}$ binaries with initial primary mass $<40 M_{\odot}$ and in all case A binaries. In our PNS model we treat case A binaries in a similar way as case B. This means in fact that we assume that also case A binaries with initial primary mass $\geq 40 M_{\odot}$ avoid RLOF. This may not be true, and deserves some attention when discussing the results of the spectral synthesis of starbursts. After more than 3 decades of extensive binary evolutionary calculations by different research teams, the following overall $\beta$ formalism for case $\mathrm{A}$ and case $\mathrm{Br}$ binaries with primary mass $<40 M_{\odot}$ emerges:

$\beta= \begin{cases}\beta_{\max } & q \geq 0.4, \mathrm{RLOF} \\ 5 \beta_{\max }(q-0.2) & 0.2 \leq q<0.4, \mathrm{RLOF} \\ 0 & q<0.2, \text { spiral-in and merging. }\end{cases}$

To calculate $\beta$ and $\beta_{\max }$, one has to solve the magnetohydrodynamic equations that describe the mass transfer, and we need a model that takes the mass accretion process into account in a realistic way. This problem is very complex and approximations are needed. An accretion model was proposed by
Neo et al. (1977) but an alternative suggestion was published by Vanbeveren \& De Loore (1994). In most cases, the conclusions related to $\beta$ depend in a critical way on the adopted accretion model and therefore, uncertainties in the latter imply uncertainties on $\beta$. Since it can be expected that $\beta=0$ for case $\mathrm{Bc}$ and case $\mathrm{C}$ binaries, one may speculate that $\beta_{\max }$ is a decreasing function of the orbital period. However, it is the scope of the present paper to illustrate possible consequences of binaries on the spectral synthesis of starbursts, for which the $\beta$ model given above is sufficient. We will present our results for different values of $\beta_{\max }$.

Of course, when matter leaves the binary we have to account for the loss of orbital angular momentum. In all our PNS results since 1997-1998, we used a formalism described by Soberman et al. (1997). Matter leaves a binary through the second Lagrangian point $L_{2}$ and settles in a circumbinary ring with radius $\eta A$ (where $A$ represents the orbital separation). A "bare minimum" for the circumbinary radius is found for $\eta \approx 1.3$, which corresponds to the distance between $L_{2}$ and the centre of mass of the binary. However as argued by Soberman et al., this ring is unstable and is likely to fragment and to fall back on the binary components. The first stable ring corresponds to $\eta \approx 2.25$. In the present paper we calculate the variation of the binary period adopting the latter value (see also Vanbeveren et al. 1998b).

An alternative formalism follows from energy considerations. Let $\mathrm{d} M_{1}$ be a small amount of mass lost by the primary during its RLOF. Suppose that part of it (i.e. $\left.(1-\beta) \mathrm{d} M_{1}\right)$ escapes from the system. To escape, the matter needs an average energy:

$\mathrm{d} E_{\text {esc }}=G \frac{M_{1}+M_{2}}{\lambda A}(1-\beta) \mathrm{d} M_{1}$

with $\lambda$ of order unity and $M_{2}$ the mass of the secondary (mass gainer). The escape energy must be supplied by the orbital energy $E$ of the system. The relation $E=-G M_{1} M_{2} / 2 A$ combined with Keplers' law yields the orbital period variation. Interestingly, the two formalisms discussed above give similar results.

It is easy to show that significant mass loss from the binary $(\beta \leq 0.5)$ is always accompanied by a large reduction of the orbital period which sometimes leads to the merging of the two components. Therefore, if PNS is computed assuming that the evolution of binaries with primary mass $<40 M_{\odot}$ is highly non-conservative, we need to consider in detail the evolution of mergers as well.

\subsection{The formation and evolution of mergers}

Binaries with initial mass ratio $q \leq 0.2$ experience a spiral-in phase during which the low mass component is dragged into the atmosphere of the most massive star and both stars merge. The final product will be a single star with a mass equal to the sum of the masses of both components, but this single merger may have a peculiar chemical composition. We consider two possibilities:

1. due to spiral-in and merging, both stars are totally mixed (Merger 1); 
2. merging is treated as genuine accretion whereby matter with a total mass equal to the mass of the low mass star is accreted onto the high mass component (Merger 2). Since our evolutionary program is able to calculate the effects of accretion, possibly accompanied by thermohaline mixing, we can estimate the evolutionary consequences of such a merger process.

Merging in binaries with mass ratio $q>0.2$ can happen as well, but in this case it is the result of the very large orbital period reduction due to a non-conservative RLOF $(\beta<1$ and/or common envelope evolution). Once a binary satisfies the conditions to merge (Vanbeveren 2001), we mix the hydrogen deficient $\mathrm{CHeB}$ mass loser remnant with the mass gainer (which is still a core hydrogen burning star in most cases) in a homogeneous way. To illustrate our procedure, consider a $30 M_{\odot}+20 M_{\odot}$ binary. Assume that $50 \%$ of the mass lost by the $30 M_{\odot}$ star during its RLOF leaves the system. At the end of RLOF, the binary consists of a $12 M_{\odot}$ hydrogen deficient remnant (WR star) and a $28 M_{\odot}$ core hydrogen burning companion. When the merging conditions are fulfilled, we mix the $12 M_{\odot}$ star with the $28 M_{\odot}$ companion. Of course, we realize that this is only an approximation. If merging happens before the end of the RLOF, less mass will be lost from the system and the merger will be more massive than assumed here. Our method should therefore be regarded as a first order approximation to the influence of mergers.

The mass gainer in a quasi-conservative binary (i.e. $\beta \approx 1$ ) may be rejuvenated by mass accretion. This means that a starburst with a population of interacting binaries, where the RLOF is accompanied by mass transfer and mass accretion, will also be rejuvenated. However, even when the RLOF is non-conservative $(\beta \ll 1)$, starbursts may be rejuvenated. A non-conservative RLOF possibly leads to the merging of the two components. Since merging implies mixing, merging leads to rejuvenation as well. This means that whatever the value of $\beta$, one expects rejuvenation of starbursts when binaries are present. Whether accretion or merging dominates obviously depends on $\beta$.

\section{The Population Spectral Synthesis (PSS) model}

PSS requires realistic theoretical spectral energy distributions (SED) or observational spectra for each star in the PNS model:

- The SEDs of hydrogen deficient CHeB stars resembling WR stars are computed using the models of Schmutz et al. (1992) which do not account for line-blanketing. The influence of the latter was studied by Smith et al. (2002). Since the grid of Smith et al. is not extended enough for the purposes of the present paper, we prefer to use the Schmutz et al. models and discuss in a qualitative way possible effects of line-blanketing.

- The SEDs of hydrogen deficient CHeB stars which do not resemble WR stars (mostly CHeB post-RLOF binary components with mass smaller than the minimum mass of WR stars, either $5 M_{\odot}$ or $8 M_{\odot}$ ) are represented by black body energy distributions. Anticipating, PNS predicts that the number of binaries with a hydrogen deficient $\mathrm{CHeB}$ component which does not resemble a WR star, is several orders of magnitude larger than the number of WR binaries. However, because the WR stars have a much larger luminosity than the non-WR hydrogen deficient $\mathrm{CHeB}$ stars, the latter class does not affect in a significant way the results of the present paper.

- The SEDs of the O type stars are interpolated from the nonLTE, line-blanketed model atmosphere grid of Smith et al. (2002); the SEDs of the other stars are calculated using the homogeneous grid of atmospheres of Lejeune et al. (1997).

- The emitted luminosities in the stellar WR lines are taken from the compilation of Schaerer \& Vacca (1998).

It is straightforward to link the Lejeune et al. SEDs to nonWR evolutionary models: we use the $T_{\text {eff }}$-Spectral Type calibration of Schmidt-Kaler (1982) and each SED is scaled according to the predicted stellar luminosity. The link between stellar evolutionary prediction and real WR stars deserves some attention. As inferred from the observed luminosities of WR stars and from the masses of the WR components in well known binaries, most of the WR stars have a mass larger than $5 M_{\odot}$ (possibly even larger than $8 M_{\odot}$, see Vanbeveren et al. 1998b). In our PNS and PSS, we consider a hydrogen deficient $\mathrm{CHeB}$ star as a WR star when its mass is larger than some minimum value. We explore the influence of this minimum in Sect. 5. To distinguish between a WNL, WNE, and WC star, we use the classical definitions as they were originally introduced by Vanbeveren \& Conti (1980), i.e. a WNL star is a WN star with hydrogen, a WNE is a WN star without hydrogen, and a WC star is a WR star with helium burning products at the surface.

The hydrostatic radii $\left(R_{\text {hydr }}\right)$ of hydrogen poor $\mathrm{CHeB}$ stars predicted by stellar evolutionary computations are on the order of $1 R_{\odot}$ in most cases, implying an evolutionary effective temperature $T_{\text {eff }}>125000 \mathrm{~K}$. However, the "hydrostatic radii" in the WR atmosphere models of Schmutz et al. (1992) are defined as the radii at optical dept $\tau \approx 10\left(R_{\text {core }}\right)$, but they are at least a factor 2 or 3 larger than $R_{\text {hydr }}$. The values resulting from the atmosphere calculations depend on a number of parameters which are uncertain (one of the main uncertainties is the velocity structure in the subsonic part of the wind, Hamann \& Schmutz 1987). As a consequence, linking evolutionary models with WR atmosphere models is not straightforward. This problem was nicely illustrated by Hamann (1994) in his Fig. 7. We will present our results assuming that the $R_{\text {core }}$ of WR atmosphere models equals $R_{\text {hydr }}$ resulting from stellar evolution, but we will also calculate models with $R_{\text {core }}$ a factor of 2 respectively a factor 3 larger than $R_{\text {hydr }}$. We would like to remind the reader that the luminosity, the lifetime, and the internal structure of a hydrogen deficient $\mathrm{CHeB}$ star do only marginally depend on how the star's atmosphere is treated, so that artificially increasing $R_{\text {core }}$ implies lower effective temperatures but does not affect its overall evolution.

The nitrogen and carbon lines that will be discussed in Sect. 5 are stellar in origin. The He II $\lambda 4686$ line may have a significant contribution from the surrounding gas. To compute the nebular line strength and continuum, we use the atomic data 
of Osterbrock (1989) and make the following assumptions concerning the physical state of the gas:

- a helium to hydrogen number density $N_{\mathrm{He}} / N_{\mathrm{H}}=0.1$;

- electron number density $n_{\mathrm{e}}=100 \mathrm{~cm}^{-3}$;

- radiation bounded nebula;

- case B recombination;

- electron temperature $T_{\mathrm{e}}=10000 \mathrm{~K}$.

\section{Observational results of starbursts useful for the present paper}

Terlevich et al. (1991) compiled the equivalent widths of the $H_{\beta}$ line (i.e. $\left.W\left(H_{\beta}\right)\right)$ of 315 extragalactic $\mathrm{H}_{\text {II }}$ regions. Guseva et al. (2000) considered a representative set of WR galaxies. Apart from $W\left(H_{\beta}\right)$, the latter authors also listed the intensity ratios $I$ (nebular He II $\lambda 4686) / I\left(H_{\beta}\right), I$ (blue bump) $/ I\left(H_{\beta}\right)$, and $I$ (red bump) $/ I\left(H_{\beta}\right)$. Of particular importance for the present paper, we notice the following features:

1. the majority of the starbursts have $W\left(H_{\beta}\right) \leq 100 \AA$;

2. although the number of observed starbursts with nearly solar metallicity is small, they never show any nebular He II 14686;

3. starbursts with small $Z$ do show nebular He II $\lambda 4686$ emission but the ratio $I$ (nebular He II $\lambda 4686) / I\left(H_{\beta}\right)$ rarely exceeds 0.03 ;

4. most WR galaxies with $Z \approx 0.02$ and with $10 \AA \leq$ $W\left(H_{\beta}\right) \leq 100 \AA$ have $I$ (blue bump) $/ I\left(H_{\beta}\right)<0.4$, whereas $I$ (red bump) $/ I\left(H_{\beta}\right)<0.08$;

5. WR galaxies with $Z \approx 0.001$ and observed $I$ (blue bump)/ $I\left(H_{\beta}\right)$ and $I($ red bump $) / I\left(H_{\beta}\right)$ ratios have $W\left(H_{\beta}\right) \approx 100 \AA$, except for a possible outlier. These galaxies have ratios $I$ (blue bump) $/ I\left(H_{\beta}\right)$ and $I$ (red bump) $/ I\left(H_{\beta}\right)$ which are smaller than 0.05 in most of the cases.

\section{Results}

As in Van Bever \& Vanbeveren (2000) we define the standard binary starburst (SBS) as follows.

- Case A binaries are treated as case Br. Our simulations allow us to conclude that PNS results are rather robust to this simplification.

- The evolution of binaries which experience a common envelope and/or spiral-in phase depends on the parameter $\alpha$ which is the amount of orbital energy which is effectively used to remove the hydrogen rich layers of the mass loser. In the SBS model we set $\alpha=1$.

- The mass loss rates of RSGs are metallicity-dependent $(\propto \sqrt{Z})$ but those of WR stars are independent from the metallicity.

- Single stars and primaries of close binaries have the same Salpeter type IMF with $M_{\min }=1 M_{\odot}$ and $M_{\max }=100 M_{\odot}$. Notice that due to the presence of the secondaries in binaries, the true IMF (single stars + primaries + secondaries) may be different from the Salpeter function (Vanbeveren 1982).
- The binary mass ratio distribution which is adopted in the SBS model is flat, whereas the binary orbital period distribution is flat in $\log P$ (periods between 1 day and 10 years) corresponding to the study of Popova et al. (1982) and Vereshchagin et al. (1987, 1988).

- The SN kick velocity distribution is $\chi^{2}$-distributed with an average kick velocity $\left\langle v_{\text {kick }}\right\rangle$ of $450 \mathrm{~km} \mathrm{~s}^{-1}$. Notice that this value determines the number of binaries that survive a $\mathrm{SN}$ explosion.

- The binary frequency at birth equals 0.8 . This percentage is consistent with the observational fact that $33 \%$ of an observed magnitude limited sample of $\mathrm{O}$ type stars are primaries of binaries with a period smaller than 100 days and a mass ratio larger than 0.2 (Garmany et al. 1980). A large massive binary frequency similar to the value used in the SBS model has also been proposed by Mason et al. (2001) and Van Rensbergen (2001).

$-\beta_{\max }=1$.

- Model "Merger 2" for binaries with mass ratio $q \leq 0.2$.

- A hydrogen deficient CHeB star (single or binary component) is considered a WR star when its mass is larger than $5 M_{\odot}$.

- The $R_{\text {core }}$ of WR stars (Sect. 3) equals the $R_{\text {hydr }}$ of hydrogen deficient $\mathrm{CHeB}$ stars resulting from evolution.

We compare the SBS results with a standard single star starburst (SSS) model. Since we want to explore spectral differences caused by binary processes, we assure that the stellar content of the SSS model is the same as in the corresponding SBS, i.e. the SSS model contains all the single stars as well as all the primaries and secondaries of the SBS model, but in the SSS model these primaries and secondaries evolve as single stars. In order to have a sufficient number of massive stars in the simulation, one has to start with a very large number of stars since we adopt a Salpeter IMF slope and $M_{\text {low }}=1 M_{\odot}$. However, the computing time can be reduced drastically as follow: the mass interval from $1 M_{\odot}$ to $100 M_{\odot}$ is divided into (for instance) 99 mass intervals of $1 M_{\odot}$. A Monte-Carlo method simulates the formation of some 100 objects in each interval. To illustrate: if the binary frequency is $80 \%$, we assume that in each mass interval (of $1 M_{\odot}$ ) there are 20 single stars and 80 primaries with a mass equal to the corresponding mass of the interval. The Monte-Carlo method distributes the 80 primaries in binaries according to the adopted period and mass ratio distributions. The evolution of each mass interval is then computed separately. In order to obtain the spectral evolution of the complete starburst, the average spectral features of each mass interval are scaled according to the Salpeter IMF. With this procedure the spectral evolution of the whole burst is simulated in a satisfactory way by considering the detailed evolution of 10000 objects only.

All our calculations presented here are performed using the population parameters summarized in Table 2. Before discussing our calculations in more detail, our simulations allow to propose the following general conclusion: the main results of the present paper do only marginally depend on the value of $\left\langle v_{\mathrm{kick}}\right\rangle$, and on how the common envelope phases and 
Table 2. The population parameters considered in the present calculations. Model a is the standard population (SBS with corresponding SSS). The other models are similar to model a but we change one or two PNS parameters.

\begin{tabular}{ll}
\hline \hline model a & standard \\
model b & Hogeveen $\phi(q)$ \\
model c & Garmany $\phi(q)$ \\
model d & minimum WR mass $=8 M_{\odot}$ \\
model e & WR mass loss $\propto \sqrt{Z}$ \\
model f & $\beta_{\max }=0.5$ \\
model g & $\beta_{\max }=0$ \\
model h & $\beta_{\max }=0$ and Merger 1 \\
\hline
\end{tabular}

spiral-in phases are treated (the value of $\alpha$ ). We will therefore not further consider the effects of these parameters.

\subsection{The $O$ and WR star population in starbursts}

\subsubsection{The O type star population}

Figures 2 and 3 illustrate the temporal variation of the number of $\mathrm{O}$ type stars. The details of the WR physics do not affect the O type star population. Models $\mathrm{d}$ and e are therefore omitted. The overall behaviour is similar for all models, independently from the initial metallicity. During the first $5 \mathrm{Myr}$ (6.5 Myr when $Z=0.001$ ) the evolution with or without binaries is the same: the number of $\mathrm{O}$ type stars decreases. At the end of this period almost all single and primary $\mathrm{O}$ type stars have disappeared. However the first binaries with primary mass less than $40 M_{\odot}$ start to interact, and a rejuvenated population with new O type stars appears, i.e. the starburst becomes rejuvenated (see also Van Bever \& Vanbeveren 1998). Notice that the rejuvenation is more pronounced when initially there are more binaries with a mass ratio close to unity (compare models $\mathrm{a}, \mathrm{b}$ and $\mathrm{c}$ ). This rejuvenation is caused mainly by two processes. The first one is the mass accretion in binaries. Obviously the effect of mass accretion decreases for lower values of $\beta_{\max }$ (models $\mathrm{f}$ and $\mathrm{g}$ ). The second rejuvenation effect is due to the merging process in binaries. As outlined in Sect. 2.3, one has to distinguish the merging process in binaries with a mass ratio $q \leq 0.2$ from the one with $q>0.2$. Merging in the latter becomes more important with smaller $\beta_{\max }$ (mass loss from the system implies orbital angular momentum loss which results in lowering of the period). When $\beta_{\max }=0$, rejuvenation is obviously not expected in a model which does not account for the merging process. The rejuvenation in the models $g$ of Figs. 2 and 3 is therefore entirely due to merging. Notice however, that this kind of rejuvenation is rather small and hardly depends on the adopted merger model for binaries with mass ratio $q<0.2$. Overall conclusion: when massive binaries are present in a starburst, rejuvenation is expected whether or not the RLOF in binaries with primary mass less than $40 M_{\odot}$ is (quasi)-conservative or not. Last but not least, after $5 \mathrm{Myr}(6.5 \mathrm{Myr}$ when $Z=0.001)$, all the $\mathrm{O}$ type stars which are still present in the burst are rejuvenated $\mathrm{O}$ type stars. One may expect them to have peculiar atmospherical chemical abundances. The pollution is due to accretion of matter lost by the primary during its RLOF or due to the merger process. Notice that these processes act together with stellar chemical mixing processes such as rotational mixing, which can dredge up CNO processed matter.

\subsubsection{The WR population}

Figure 4 illustrates the evolution of the number of stars in the three WR subtypes (i.e. WNL, WNE and WC). We always compare a simulation containing binaries with the corresponding single star starburst. We only retain the models which differ most from the standard model a at solar metallicity, i.e. model d $(Z=0.02)$, model a $(Z=0.001)$, model e $(Z=0.001)$ and model g $(Z=0.001)$. With another initial mass ratio distribution or with an alternative treatment of the merger process, the results change quantitatively, but compared to the SBS model the overall differences are not significant.

The main influence of the presence of binaries in a starburst on the WR population is a prolongation of the WR phase of the burst. Obviously, this prolongation is less pronounced when the minimum WR mass is larger. This is illustrated by the $Z=0.02$ results when model a is compared to model d.

The WR phase duration is also influenced by the value of $\beta_{\max }$ (see Fig. 4, at $Z=0.001$, compare model a and model g). Since $\beta_{\max }=0$ in the latter, the small prolongation is entirely due to the merging process in binaries.

Model e, at $Z=0.001$, illustrates the effect of a metallicitydependent WR stellar wind mass loss rate. Compared to the WR mass loss rates in the standard model (i.e. not $Z$ dependent), a $Z$-dependent WR wind is considerably smaller in low metallicity regions. This means that it takes longer before all the CNO enhanced layers are removed and $3 \alpha$ products appear at the surface, i.e. the WN phase of the starburst is lengthens and its WC phase shortens.

Due to our adopted LBV scenario, starbursts with or without binaries which are younger than the evolutionary lifetime of a $40 M_{\odot}$ star have the same WR population. Since we assume that this LBV scenario does not depend on metallicity, the same holds for our $Z=0.001$ simulations. Notice, however, that this would be entirely different if the LBV stellar wind mass loss was metallicity-dependent as well.

With our preferred RSG stellar wind mass loss formalism, stellar evolution in combination with PNS predicts that the population of WR single stars resulting from stars with an initial mass $<40 M_{\odot}$ in $Z=0.02$ starbursts, evolves in a similar way as the population of WR primaries with progenitors with initial mass smaller than $40 M_{\odot}$. Notice that the foregoing would not be true if evolutionary calculations of single stars are adopted with lower RSG mass loss rates like those of Schaller et al. (1992), as used by Leitherer et al. (1999) and by Schaerer \& Vacca (1998). Since we adopt metallicity-dependent RSG mass loss rates in the evolutionary tracks, the single WR star population and the binary WR star population is obviously entirely different in the case $Z=0.001$. 


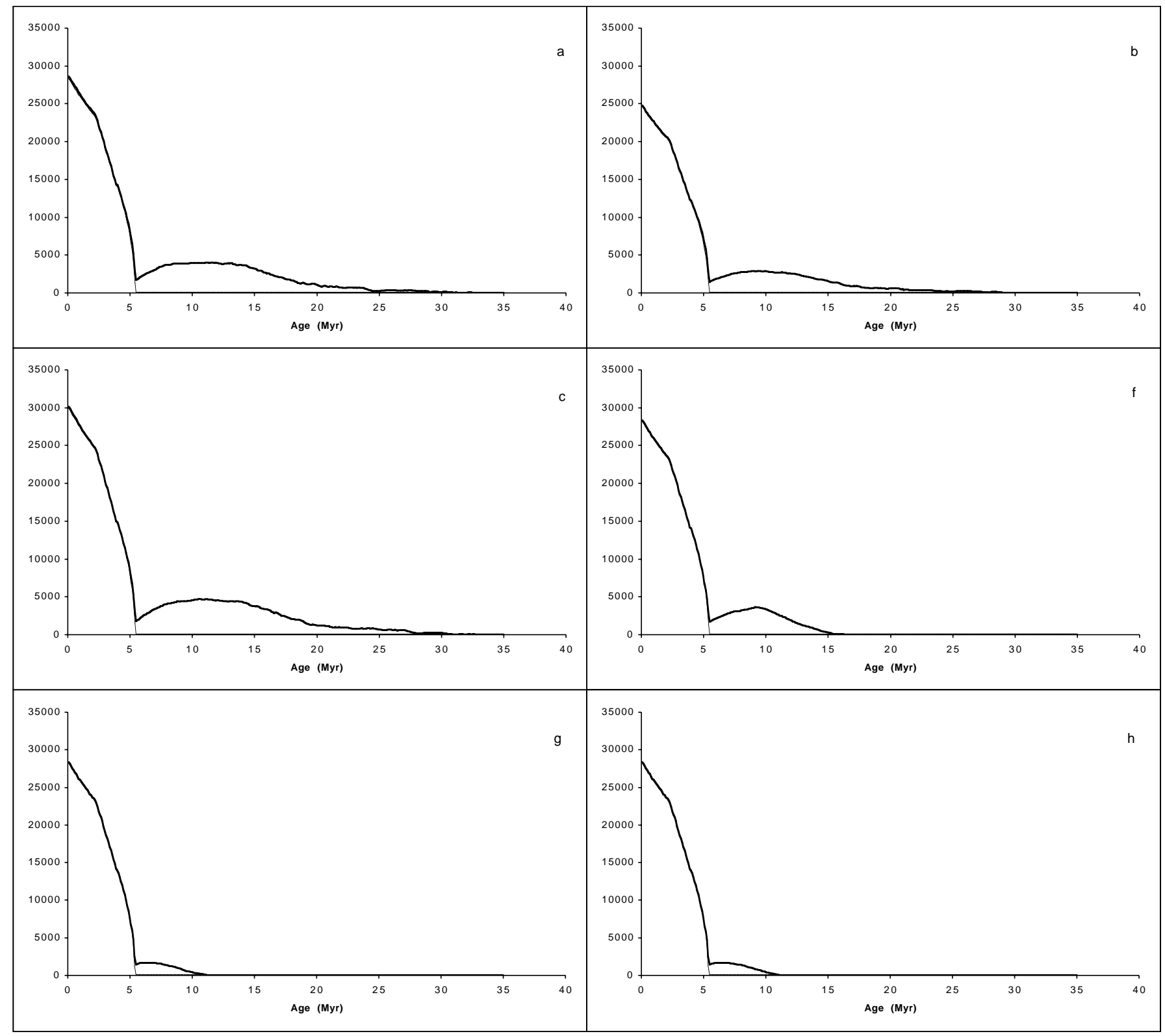

Fig. 2. The variation of the number of $\mathrm{O}$ type stars as a function of time (in Myr) for $Z=0.02$, for some of the models from Table 2 . We only show the models which differ most from the SBS model. Thick lines hold for starsbursts with binaries, thin lines for starbursts with single stars only.

\subsection{The evolution of the nebular $\mathrm{H}_{\beta}$ line}

Figure 5 shows the temporal variation of $W\left(H_{\beta}\right)$ for some of the models of Table 2. Similarly as in the previous sections, we selected only the models which differ most from the corresponding standard model. These results can be considered as an extension (and an update since we now use the model atmospheres of O type stars of Smith et al. 2002) of the calculations of Van Bever et al. (1999) who only considered solar metallicity starbursts, and did not account for mergers. The starburst rejuvenation processes discussed in Sect. 5.1 are clearly reflected in the figure, i.e. after about $4 \mathrm{Myr}$ rejuvenated $\mathrm{O}$ type stars appear due to mass accretion and merging of binaries with primary mass $<40 M_{\odot}$ and they produce new $\mathrm{O}$ stars which create a plateau in the $W\left(H_{\beta}\right)$ curve at around $100 \AA(150 \AA$ for low metallicity starbursts) which lasts for several million years. This means that binaries make age determinations ambiguous when the starburst has a $W\left(H_{\beta}\right) \approx 100 \AA$ or so. Notice however, that the rejuvenation is most extreme when $\beta_{\max }=1$. The observations (see Sect. 4) reveal that the majority of the known $\mathrm{H}_{\text {II }}$ and/or WR galaxies have a $W\left(H_{\beta}\right)$ around $100 \AA$ or smaller, and could therefore be affected by the age ambiguity discussed above.

Our results illustrate once more the well known discrepancy that theoretical simulations of starbursts (where star formation is (almost) instantaneous) predict too many candidates with very large $W\left(H_{\beta}\right)$. The reason may be related to the formation history of massive stars. The results discussed above 


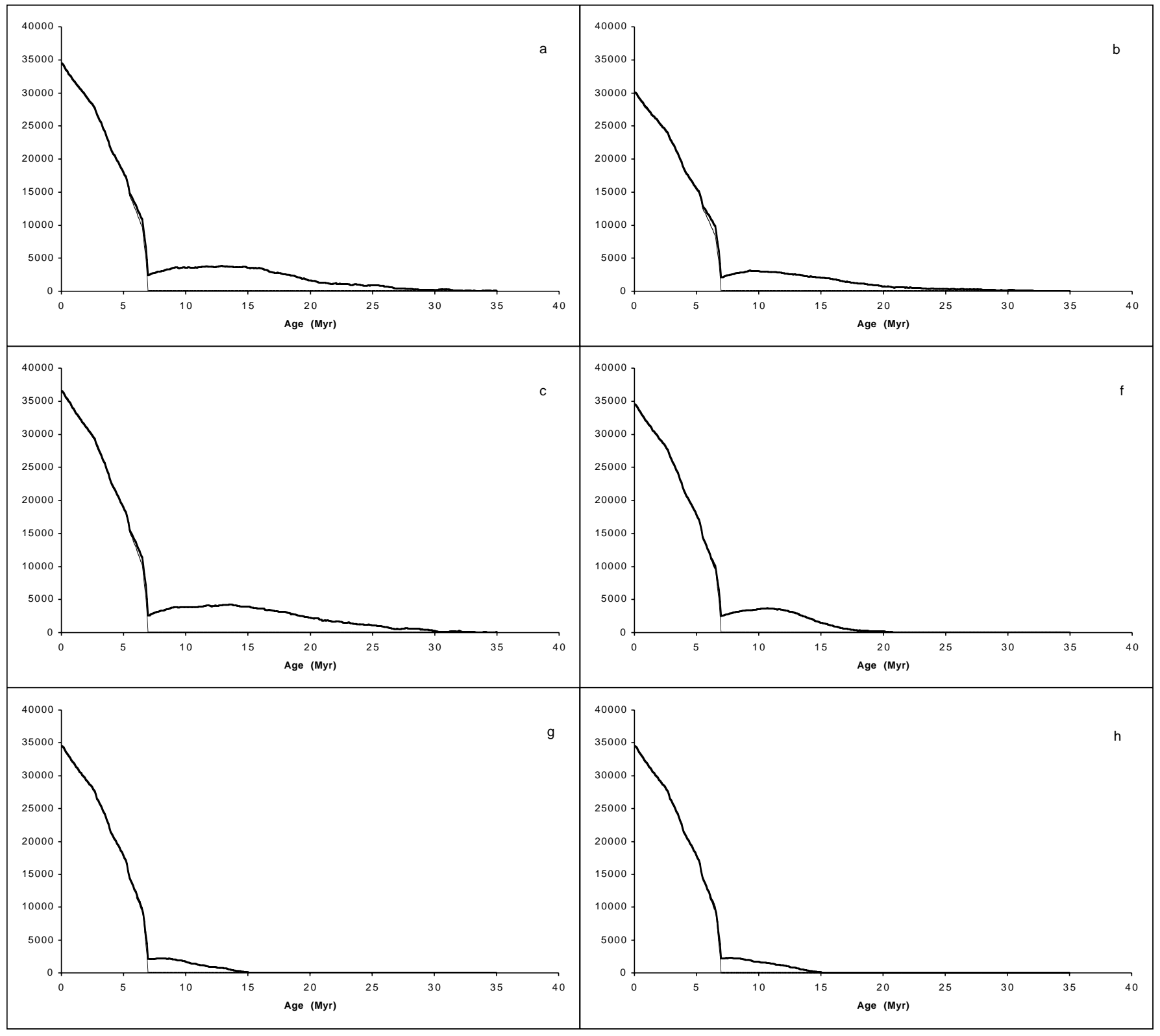

Fig. 3. As Fig. 2 but at $Z=0.001$.

apply when the formation timescale of massive stars is small compared to their core hydrogen burning nuclear timescale. However, an alternative scenario was suggested by Bernasconi $\&$ Maeder (1996). From the protostellar molecular cloud, a lower mass star forms first. But when radiation pressure becomes important, further accretion on the star is slowed down. If the accretion timescale is similar to the nuclear timescale, a significant part of the core hydrogen burning phase may happen during the accretion phase, when the star is still in the molecular cloud cocoon. It is easy to understand that when this model is included in the simulations, the predicted number of starbursts with large $W\left(H_{\beta}\right)$ will be smaller, i.e. the lack of a large number of starbursts with large $W\left(H_{\beta}\right)$ may be related to the formation process of massive stars.
The calculations presented here were performed by assuming $R_{\text {core }}=R_{\text {hydr }}$ for WR stars. However, the rejuvenation effect seen in the evolution of $W\left(H_{\beta}\right)$ is caused mainly by the number of rejuvenated $\mathrm{O}$ type stars. Therefore, as far as the rejuvenation effects on $W\left(H_{\beta}\right)$ are concerned, our conclusions do not significantly depend on the detailed physics of the WR atmospheres.

In our simulations we treated case A binaries in a similar way as case $\mathrm{Br}$ systems. For case A systems with a primary mass below $40 M_{\odot}$ this is more than sufficient. We made some test calculations of a starburst whose case A binaries with primary mass larger than $40 M_{\odot}$ avoid the LBV phase and evolve through a conservative RLOF. The results are obvious, i.e. compared to our simulations presented in Fig. 5, the 
J. Van Bever and D. Vanbeveren: The effects of binaries on Wolf-Rayet spectral features in starbursts

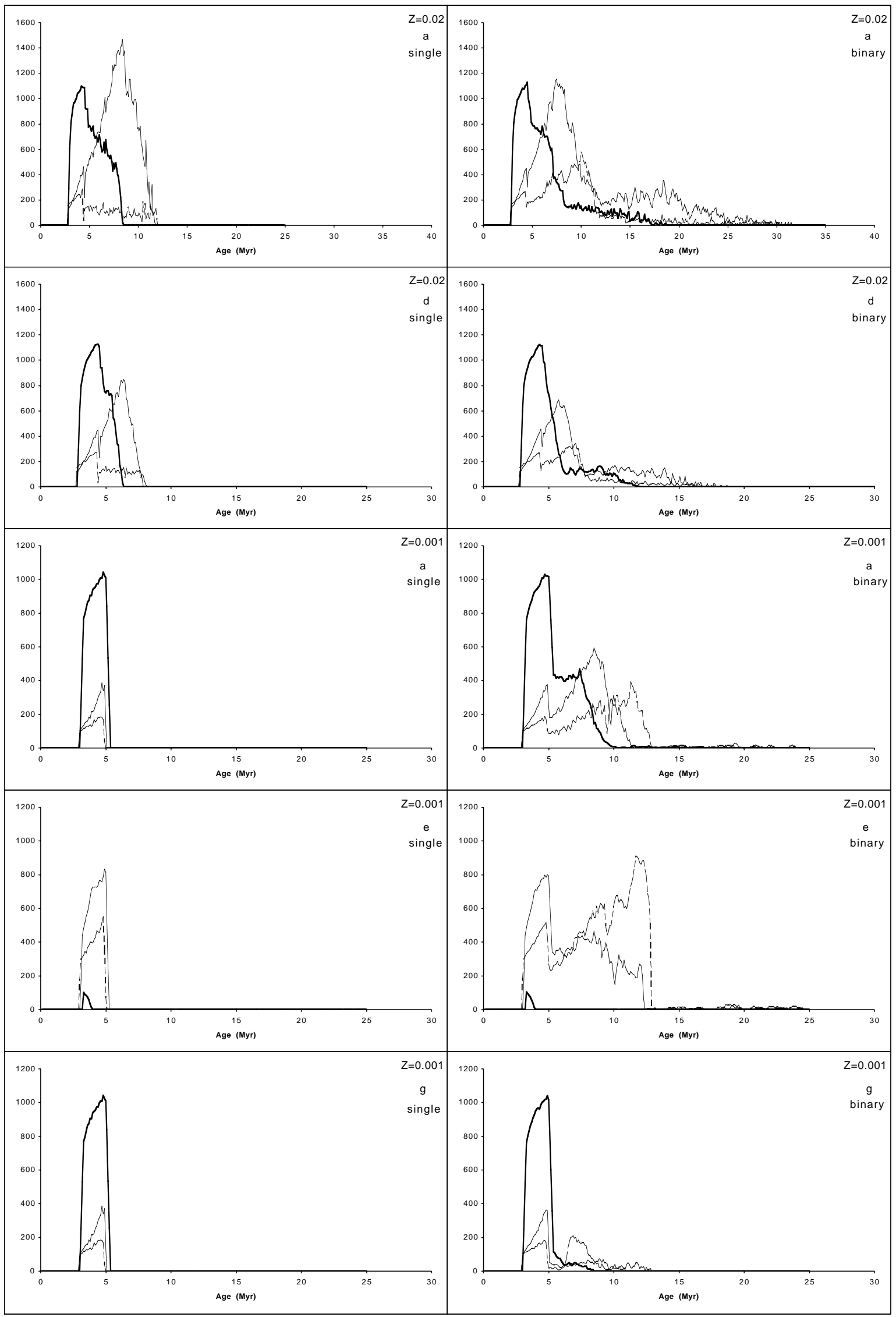

Fig. 4. The temporal evolution of the number of WNL, WNE and WC stars. Thick lines are WC stars, thin lines denote WNE stars, and the dashed line corresponds to the WNL stars. The figures with the label "single" correspond to starbursts with single star only, those with the label "binaries" correspond to models referred to in Table 2. 


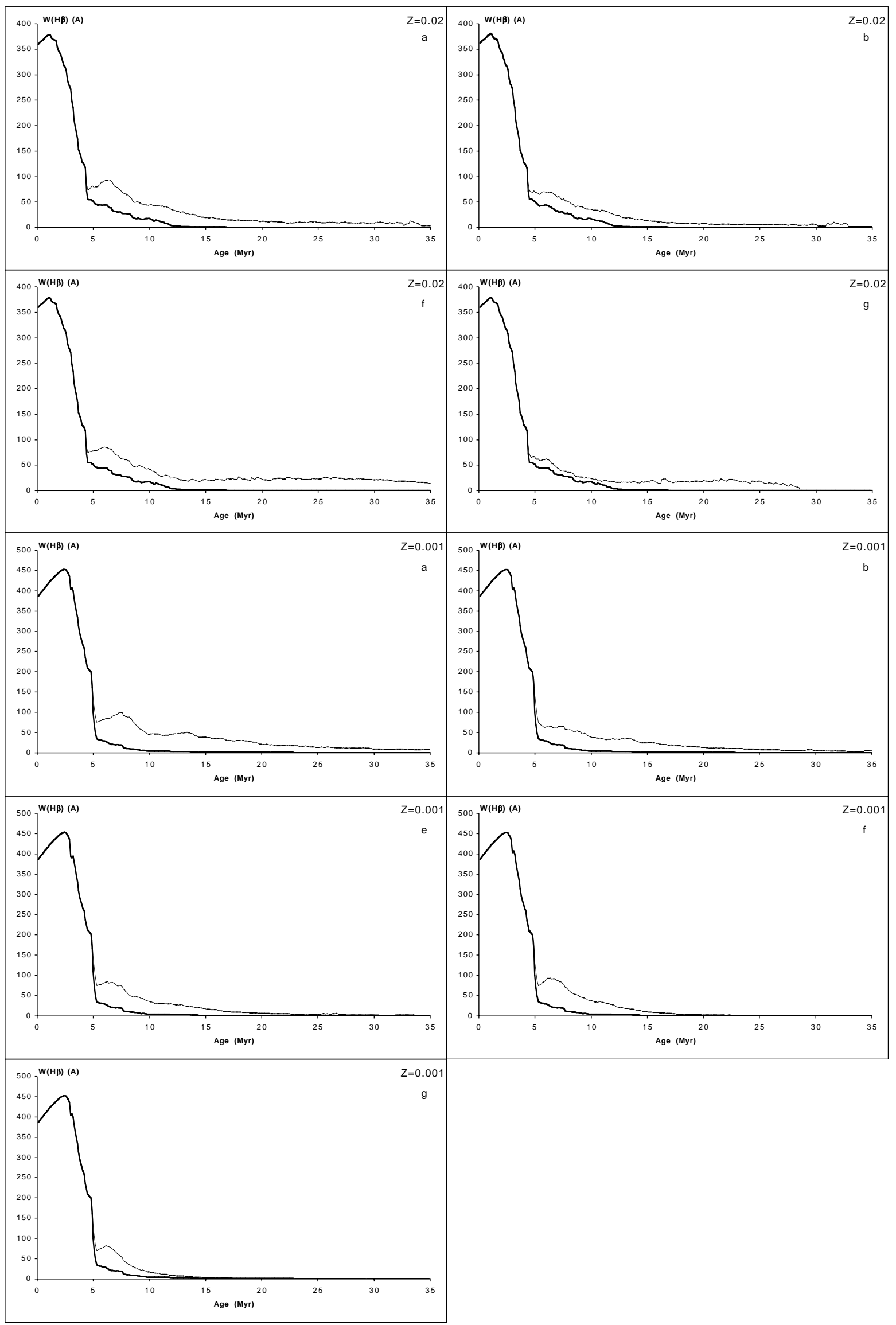

Fig. 5. The variation of $W\left(H_{\beta}\right)$ as a function of time (in Myr) for some of the models of Table 2 . The thin lines correspond to the binary model. The thick lines corresponds to the corresponding single star model. 


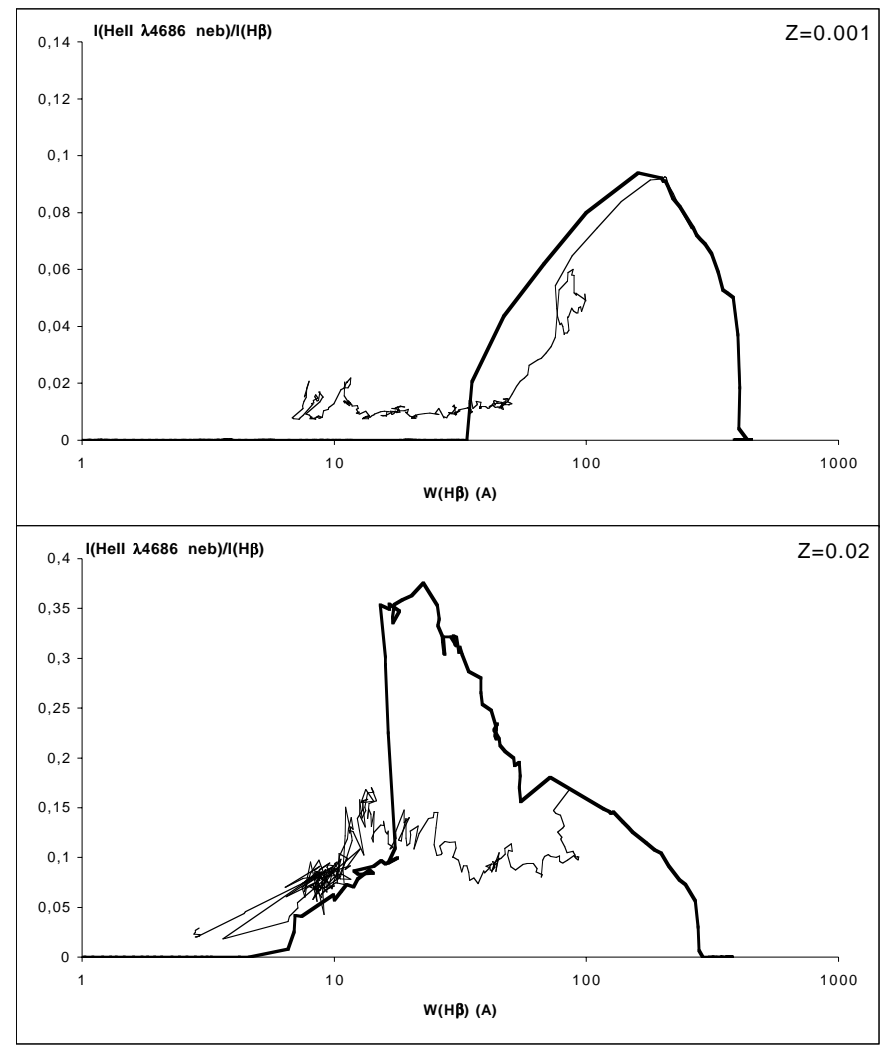

Fig. 6. The theoretically predicted evolution of the intensity ratio $I($ nebular He II 24686$) / I\left(H_{\beta}\right)$ as a function of $W\left(H_{\beta}\right)$. Thick lines denote the SSS model while thin lines correspond to the SBS model.

rejuvenation effects are more pronounced and the equivalent widths are increased, but the effect is at most $10-15 \%$.

\subsection{The evolution of the nebular He II $\lambda 4686$ line}

Nebular He II $\lambda 4686$ emision is obviously associated with hot stars which have a significant $\mathrm{He}^{+}$ionizing flux. Here we distinguish two types of stars which are hot enough: the luminous WR stars (single and binary components) and all the post-RLOF primaries with mass smaller than the minimum WR mass. Notice however, that the latter class of objects never contribute significantly to the nebular He II $\lambda 4686$ emission in starbursts. Figure 6 illustrates the evolution of the intensity ratio $I$ (nebular He II $\lambda 4686) / I\left(H_{\beta}\right)$. We restrict ourselves to the SBS and SSS simulations for $Z=0.02$ and $Z=0.001$ since other combinations of the PNS parameters predict the same range of values for the intensity ratio. Remember that in the standard models we link the $R_{\text {core }}$ of the WR atmospheres of Schmutz et al. (1992) with the $R_{\text {hydr }}$ predicted by stellar evolution. We conclude that compared to observations, these theoretical simulations predict too much nebular He II $\lambda 4686$ emision. Interestingly, by interpreting carefully the analysis of Smith et al. (2002), it follows that the discrepancy remains if in the standard starburst models, WR atmospheres are used with lineblanketing.

The adopted link between $R_{\text {hydr }}$ and $R_{\text {core }}$ (see Sect. 3 ) is crucial in the analysis of the nebular He II $\lambda 4686$ line. In Fig. 7 we compare the SBS models adopting different values
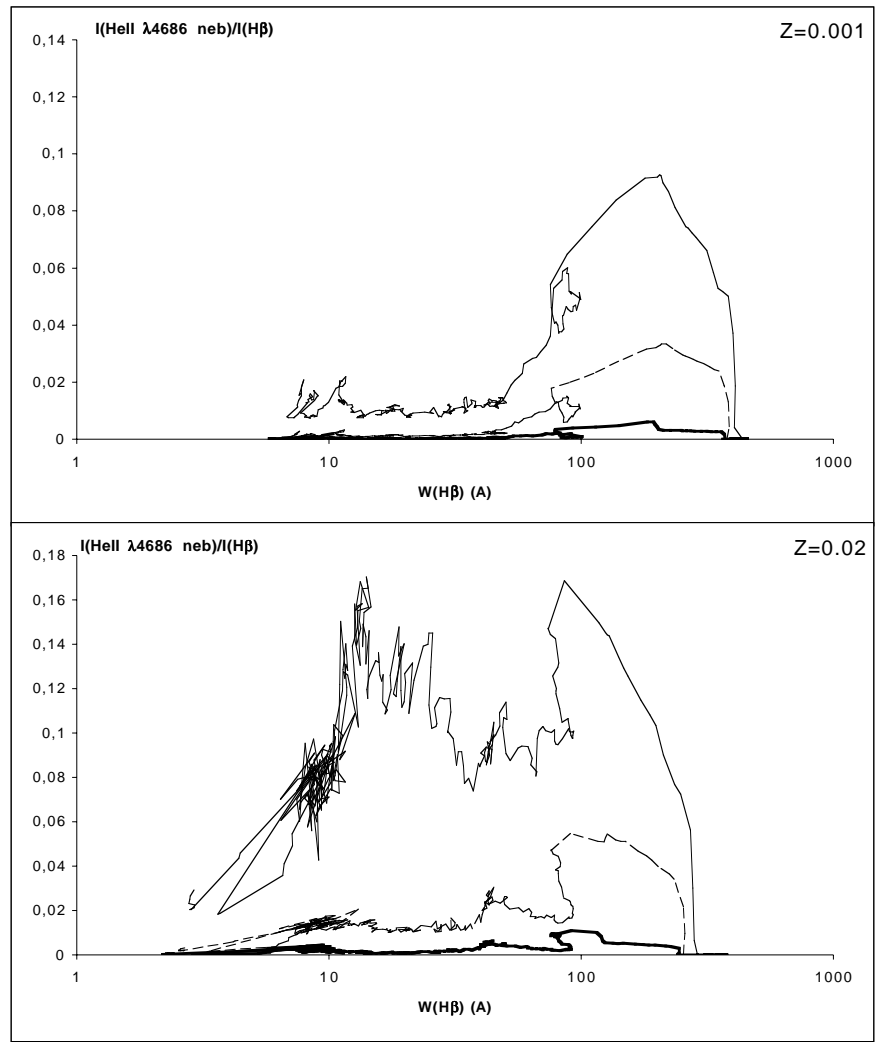

Fig. 7. The effect of different core radii of WR stars on the theoretically predicted evolution of the intensity ratio $I$ (nebular He II 14686) $/ I\left(H_{\beta}\right)$ as a function of $W\left(H_{\beta}\right)$. Only the SBS model is shown. The full thin line corresponds to the case where $R_{\text {core }}=R_{\text {hydr }}$, the dashed line to $R_{\text {core }}=2 R_{\text {hydr }}$ and the thick line to $R_{\text {core }}=3 R_{\text {hydr }}$.

for $R_{\text {core }}$ (twice and three times $R_{\text {hydr }}$ ). As can be noticed, the effect is overwhelming and largely overtakes the effects of other PNS parameters and/or the effect of line-blanketing. The observations (points 2 and 3 of Sect. 4) reveal small (at small $Z$ ) or no (at solar metallicity) nebular He II $\lambda 4686$ emission. If this effect is linked to the radius effect, one could be tempted to conclude that the WR star atmospheres have core radii which are a factor of 2 to 3 larger than the hydrostatic evolutionary radii. At least to our knowledge, there is no physics that explains this difference, which raises the question: is there something missing in the stellar structure equations, or is there something missing in the theory of WR atmospheres? A more detailed interpretation of the nebular He II $\lambda 4686$ emission has to await an answer on the foregoing question.

\subsection{The blue and red WR bump}

In Figs. 8 and 9 we plot the intensity ratio $I$ (blue bump) $/ I\left(H_{\beta}\right)$ and $I\left(\right.$ red bump) $/ I\left(H_{\beta}\right)$ as function of $W\left(H_{\beta}\right)$. Again we only consider the models whose results deviate significantly from the standard model at $Z=0.02$ and at $Z=0.001$. Also here, the effect of binaries is very significant in starbursts with $W\left(H_{\beta}\right) \leq$ $100 \AA$. The low intensity ratios observed at $Z=0.02$ (Sect. 4) are better reproduced with binaries.

The figures also illustrate that for starbursts with small $Z$ a metallicity-dependent WR mass loss rate formalism is of 


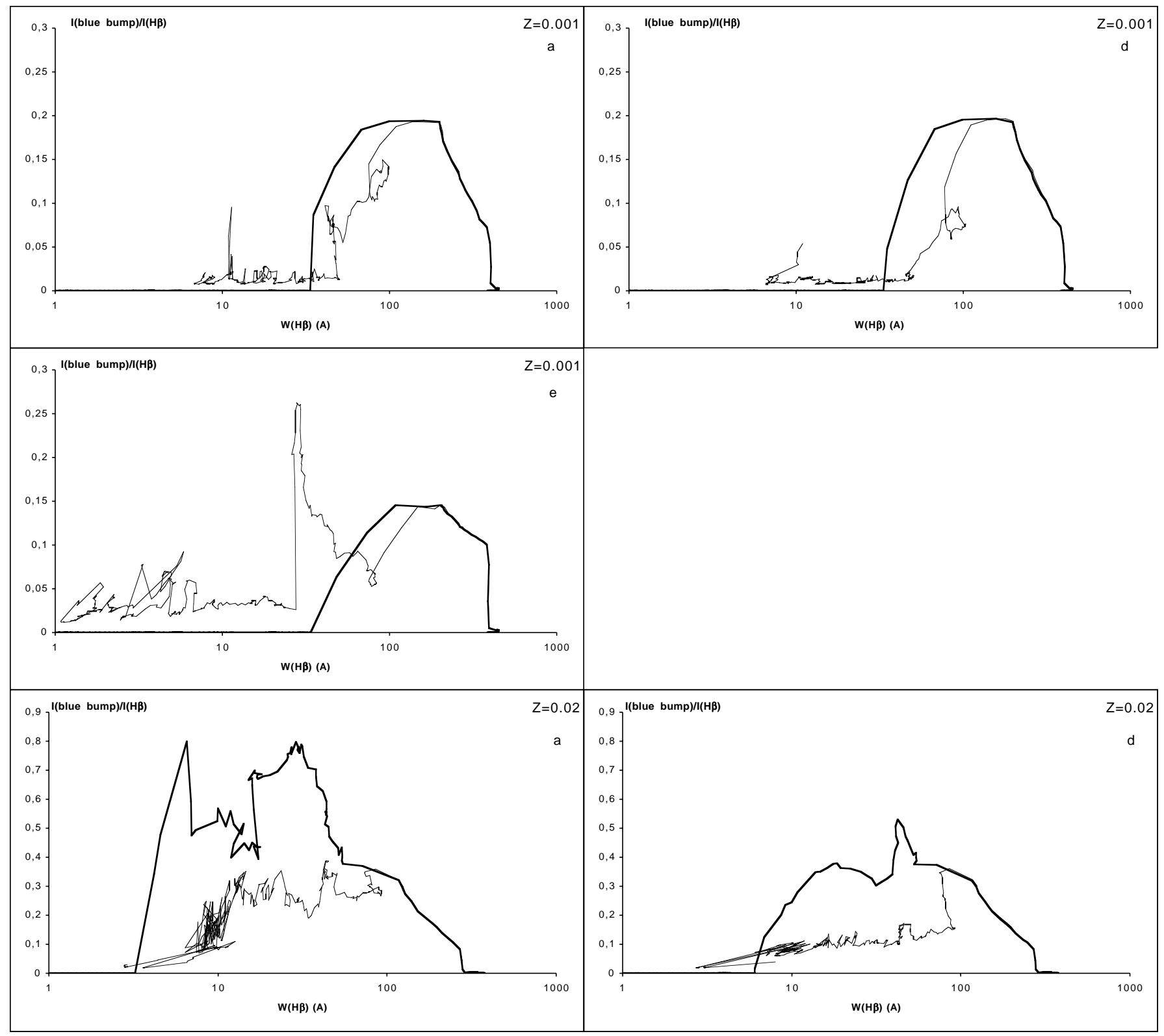

Fig. 8. The predicted evolution of $I$ (blue bump) $/ I\left(H_{\beta}\right)$ as a function of $W\left(H_{\beta}\right)$ for some of the models of Table 2. The lines have the same meaning as in Fig. 6.

crucial importance for theoretical simulation. Observational point 5 (Sect. 4) is best reproduced by a model with a significant number of binaries, and a WR mass loss rate which is metallicity-dependent. A similar conclusion holds when one compares the observed and theoretically predicted WC/WN number ratio in regions where star formation is continuous in time (Vanbeveren 2001). Notice that in a number of studies published in the past (e.g. Massey 1999) the observed variation as a function of metallicity of the $\mathrm{WC} / \mathrm{WN}$ ratio was attributed to the $Z$-dependency of the stellar wind mass loss rate during the RSG. However, since binary components rarely experience a RSG phase, this interpretation is valid ONLY if binaries can be completely neglected. The latter is questionable especially if one considers the fact that in the SMC many WR stars are close binary members (Foellmi \& Moffat 2001, 2003).

\section{Overall conclusions}

In the present paper we have investigated in detail the effects of binaries on the theoretical simulation of the spectral synthesis of starbursts, with special emphasis on the WR features. We conclude:

- Binaries play a very significant role in the simulations of WR galaxies that calculate $W\left(H_{\beta}\right)$, intensity ratios $I($ nebular He II $\lambda 4686) / I\left(H_{\beta}\right), I$ (blue bump) $/ I\left(H_{\beta}\right)$, and $I\left(\right.$ red bump) $/ I\left(H_{\beta}\right)$; the binary processes which are dominant are the Roche lobe overflow, the mass transfer efficiency, and the merger rate.

- The results for the $I($ nebular He II $\lambda 4686) / I\left(H_{\beta}\right)$ ratio are dominated by the uncertainty in the $R_{\text {core }}$ of theoretical WR atmospheres which is directly related to the 


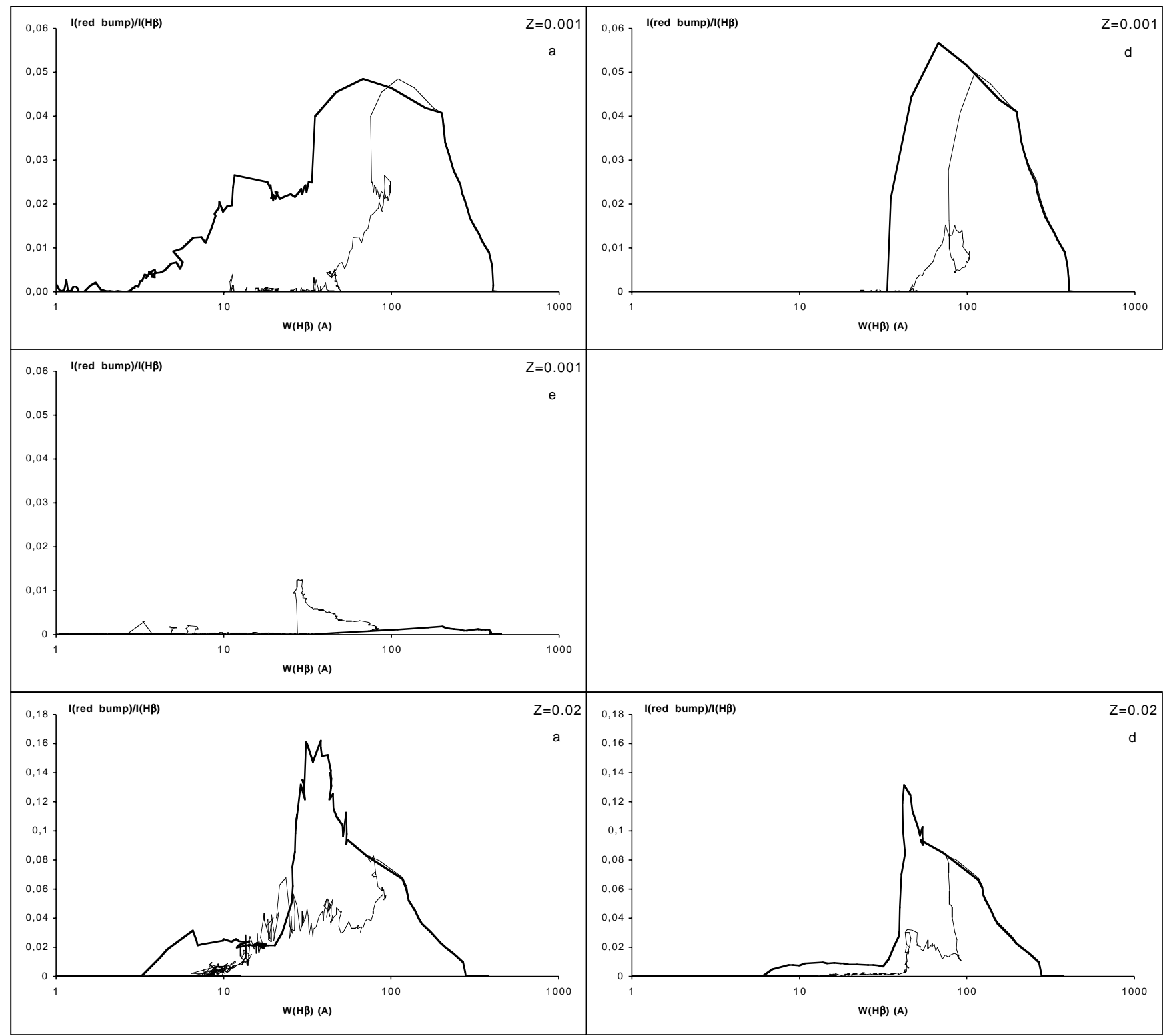

Fig. 9. The predicted evolution of I(red WR bump)/I(H $\left.H_{\beta}\right)$ as a function of $W\left(H_{\beta}\right)$ for some of the models of Table 2 . The lines have the same meaning as in Fig. 6.

uncertainty in the structure of the subsonic velocity region of the WR wind.

- The theoretical simulations of low metallicity starbursts critically depend on whether or not the WR mass loss rate formalism is metallicity-dependent. The observations are best reproduced by a model with a significant number of interacting binaries and a metallicity-dependent WR wind.

- Overall: theoretical simulation is able to reproduce qualitatively the spectral observations of starbursts and we are getting closer to a more realistic model with promising results.

Acknowledgements. We thank the unknown referee for the very valuable remarks and suggestions which improved the present paper considerably.

\section{Appendix A: The CHeB evolution of massive stars}

The evolution of the WR spectral features in starbursts critically depends on the WR mass loss rates and their effects on massive star evolution. As noted in Sect. 2.1.3, since 1997 we use evolutionary models in which the WR mass loss rates obey Eq. (1). As illustrated in Fig. A.1, the final mass prior to core collapse in our computations is much larger compared to pre1997 evolutionary tracks published by different research teams. With our preferred WR mass loss rate formalism, Galactic stars with initial mass between $40 M_{\odot}$ and $100 M_{\odot}$ end their life with a mass between $10 M_{\odot}$ and $20 M_{\odot}$ corresponding to $\mathrm{CO}$ cores with a mass between $5 M_{\odot}$ and $15 M_{\odot}$. These results are particularly important when considering the observed masses of black hole candidates in X-ray binaries. Figure A.1 also shows 


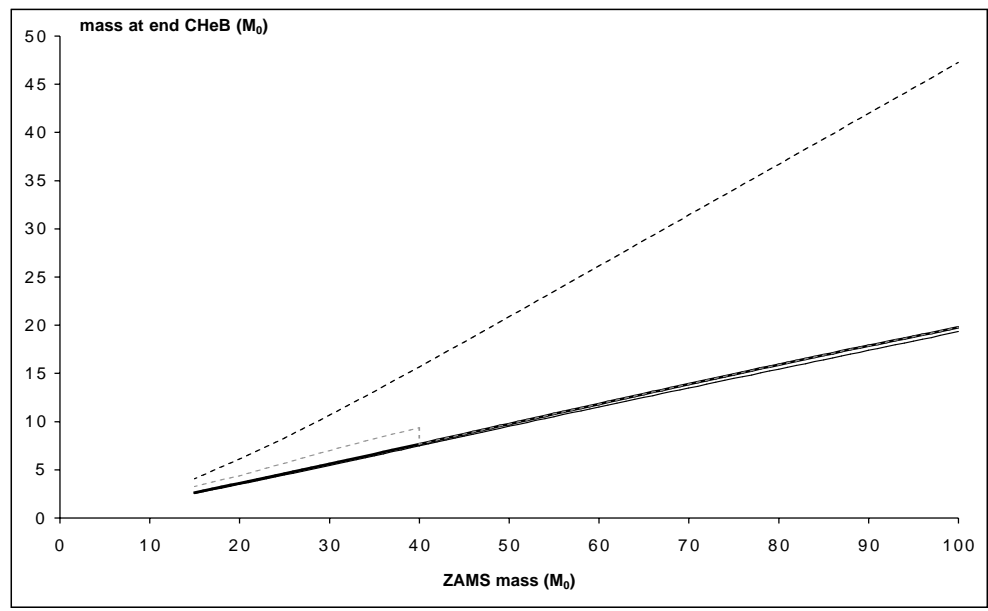

Fig. A.1. The pre-supernova mass of our models as a function of their initial mass. The dashed line corresponds to primaries of case $\mathrm{B}$ binaries at $Z=0.001$ whose WR mass loss rate $\propto \sqrt{Z}$. The full thin line corresponds to $Z=0.001$ models computed using the same WR mass loss rates as at $Z=0.02$. The full thick line holds for primaries of case $B$ binaries at $Z=0.02$. The grey dashed line is for single stars at $Z=0.02$ (note that we assume that case $\mathrm{Br}$ primaries with initial mass $\geq 40 M_{\odot}$ evolve according to the LBV scenario summarized in Sect. 2).

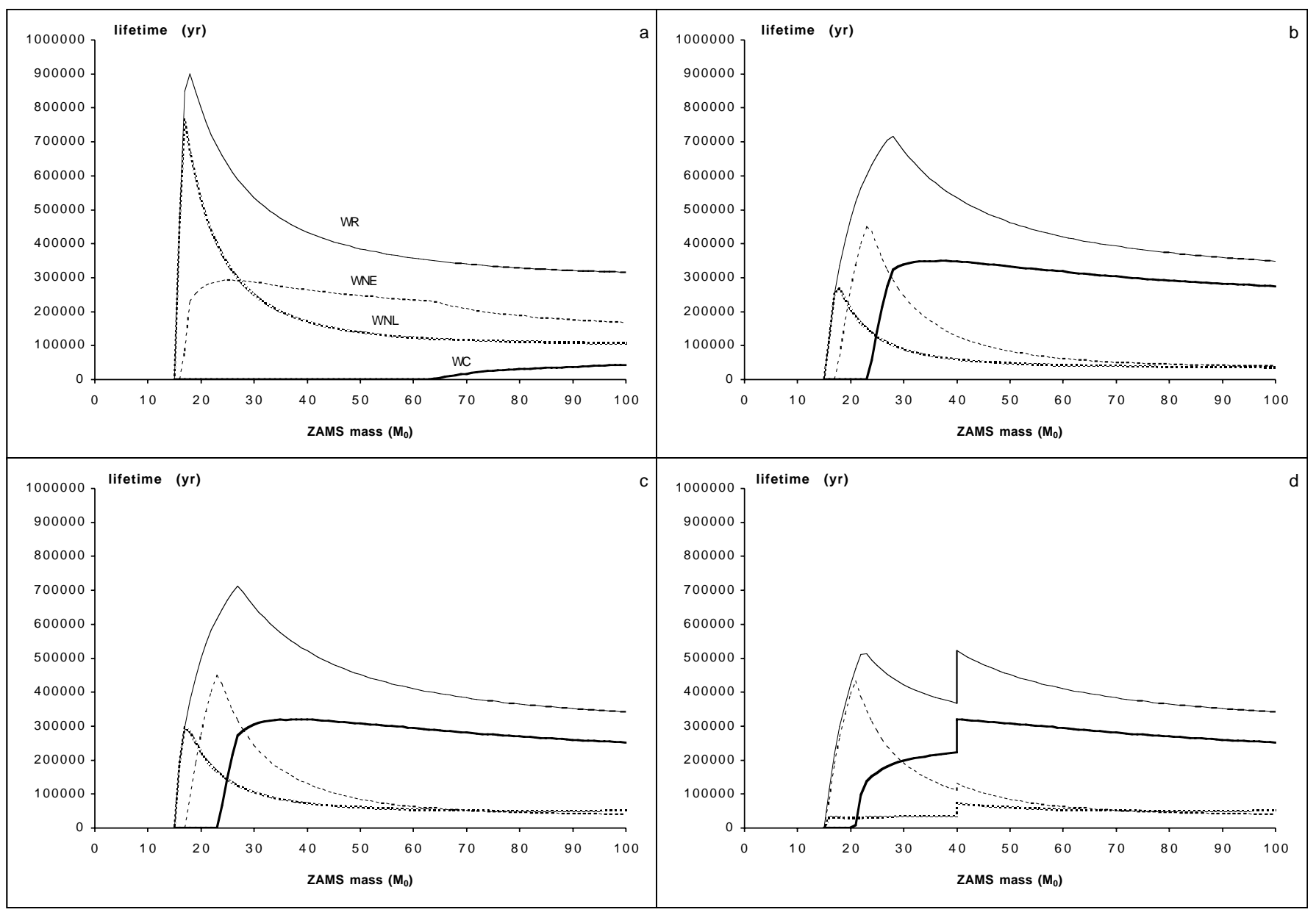

Fig. A.2. The WNL, WNE, WC and WR lifetime as a function of the initial mass for a) primaries of case $\mathrm{B}$ binaries at $Z=0.001$ with a WR mass loss rate $\propto \sqrt{Z}$, b) primaries of case B binaries at $Z=0.001$ using solar metallicity WR mass loss rates, c) primaries of case B binaries at $Z=0.02$, d) single stars at $Z=0.02$ (note that for initial masses $\geq 40 M_{\odot}$, the single star WR lifetimes equal those of binaries due to the adopted LBV scenario. The minimum WR mass is $5 M_{\odot}$ ).

the final masses of massive stars in the SMC (i.e. $Z_{\mathrm{SMC}}=$ $\left.0.1 Z_{\odot}\right)$, assuming that the WR mass loss rate is metallicitydependent $(\propto \sqrt{Z})$. Notice that, compared to objects at solar metallicity, the masses of the black hole components in X-ray binaries in low metallicity regions could be considerably larger.
Figures A.2 and A.3 focuse on the lifetimes of primaries and single stars spent as a WNL, WNE, and WC star. Notice that the lifetimes depend critically on the adopted minimum WR mass, the metallicity, as well as on the metallicity-dependence of the WR mass loss rate. 


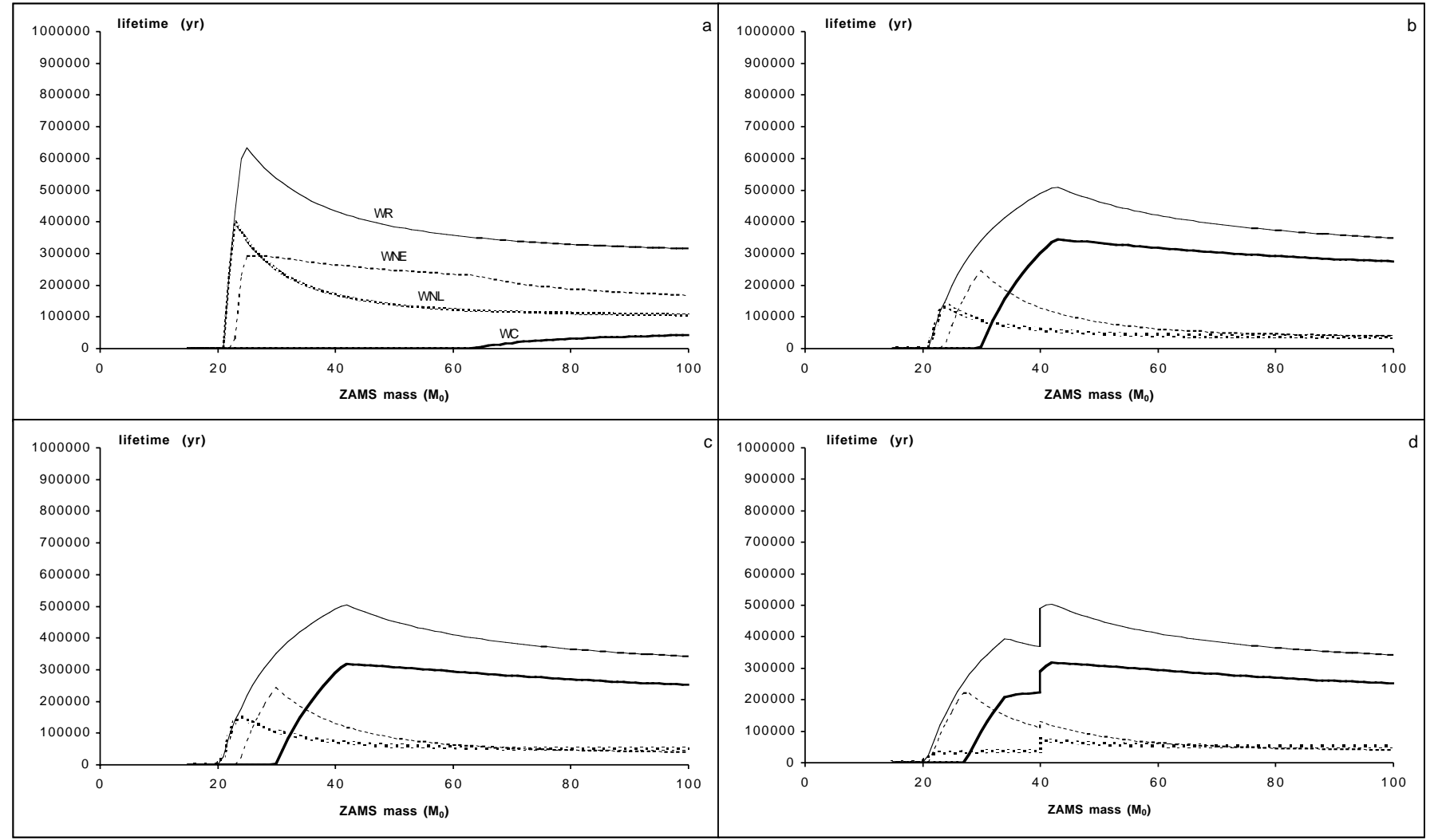

Fig. A.3. as Fig. A.2 but using a minimum WR mass of $8 M_{\odot}$.

\section{References}

Bernasconi, P. A., \& Maeder, A. 1996, A\&A, 307, 829

Conti, P. S. 2000, PASP, 112, 1413

De Marco, O., Schmutz, W., Crowther, P. A., et al. 2000, A\&A, 358, 187

Dessart, L., Crowther, P. A., Hillier, D. J., et al. 2000, MNRAS, 315, 407

Dionne, D. 1999, in 193rd IAU Symp., Wolf-Rayet Phenomena in Massive Stars and Starburst Galaxies, ed. K. A. van der Hucht, G. Koenigsberger, \& P. R. J. Eenens (San Francisco: Astronomical Society of the Pacific), 596

Foellmi, C., \& Moffat, A. F. J. 2001, in The Influence of Binaries on Stellar Population Studies, ed. D. Vanbeveren (Dordrecht: Kluwer Academic Publishers), 157

Foellmi, C., \& Moffat, A. F. J. 2003, in A Massive Star Odyssee: from Main Sequence to Supernova, in press

Garmany, C. D., Conti, P. S., \& Massey, P. 1980, ApJ, 242, 1063

Guseva, N. G., Izotov, Y. I., \& Thuan, T. X. 2000, ApJ, 531, 776

Hamann, W.-R., Koesterke, L., \& Wessolowski, U. 1995, A\&A, 299, 151

Hamann, W.-R., \& Koesterke, L. 1998, A\&A, 335, 1003

Hamann, W.-R., \& Koesterke, L. 2000, A\&A, 360, 647

Hamann, W.-R. 1994, Space Sci. Rev., 66, 237

Hamann, W.-R., \& Schmutz, W. 1987, A\&A, 174, 173

Hillier, D. J., \& Miller, D. 1999, ApJ, 519, 354

Hillier, D. J. 1996, in WR stars in the Framework of Stellar Evolution, ed. J. M. Vreux, A. Detal, D. Fraipont-Caro, E. Gosset, \& G. Rauw (Université de Liège), 509

Hogeveen, S. 1991, Ph.D. Thesis, University of Amsterdam Hogeveen, S. 1992, Ap\&SS, 196, 299

Humphreys, R. M., \& Davidson, K. 1994, PASP, 106, 1025

Humphreys, R. M., \& McElroy, D. B. 1984, ApJ, 284, 565
Jura, M. 1987, ApJ, 313, 743

Langer, N. 1989, A\&A, 210, 93

Leitherer, C., Schaerer, D., Goldader, J. D., et al. 1999, ApJS, 123, 3

Lejeune, T., Cuisenier, F., \& Buser, R. 1997, A\&AS, 125, 229

Lorimer, D. R., Bailes, M., \& Harrison, P. A. 1997, MNRAS, 289, 592

Massey, P. 1999, in 193rd IAU Symp., Wolf-Rayet Phenomena in Massive Stars and Starburst Galaxies, ed. K. A. van der Hucht, G. Koenigsberger, \& P. R. J. Eenens (San Francisco: Astronomical Society of the Pacific), 429

Mason, B. D., Gies, D. R., \& Hartkopf, W. I. 2001, in The Influence of Binaries on Stellar Population Studies, ed. D. Vanbeveren (Dordrecht: Kluwer Academic Publishers), 37

Mermilliod, J.-C. 2001, in The Influence of Binaries on Stellar Population Studies, ed. D. Vanbeveren (Dordrecht: Kluwer Academic Publishers), 3

Moffat, A. F. J. 1996, in WR stars in the Framework of Stellar Evolution, ed. J. M. Vreux, A. Detal, D. Fraipont-Caro, E. Gosset, \& G. Rauw (Université de Liège), 199

Morris, P. W., van der Hucht, K. A., Crowther, P. A., et al. 2000, A\&A, 353,624

Neo, S., Miyaji, S., Nomoto, K., \& Sugimoto, D. 1977, PASJ, 29, 249

Nugis, T., \& Lamers, H. J. G. L. M. 2000, A\&A, 360, 227

Nugis, T., Willis, A. J., \& Crowther, P. A. 1998, A\&A, 333, 956

Osterbrock, D. E. 1989, in Astrophysics of Gaseous Nebulae and Active Galactic Nuclei (University Science Books, California)

Popova, E. I., Tutukov, A. V., \& Yungelson, L. R. 1982, Astron. Space Sci., 88,55

Reid, N., Tinney, C., \& Mould, J. 1990, ApJ, 348, 98

Salpeter, E. 1955, ApJ, 121, 161

Schaerer, D., \& Vacca, W. D. 1998, ApJ, 497, 618

Schaller, G., Schaerer, D., Meynet, G., \& Maeder, A. 1992, A\&AS, 96, 269 
Schmidt-Kaler, T. 1982, in Landolt-Börnstein, New Series, Group VI, Vol. 2b, ed. K. Schaifers, \& H. H. Voigt (Berlin: Springer), 1

Schmutz, W., Geballe, T., \& Schild, H. 1996, A\&A, 311, L25

Schmutz, W., Leitherer, C., \& Gruenwald, R. 1992, PASP, 104, 1164

Schmutz, W. 1996, in WR stars in the Framework of Stellar Evolution, ed. J. M. Vreux, A. Detal, D. Fraipont-Caro, E. Gosset, \& G. Rauw (Université de Liège), 553

Schmutz, W. 1997, A\&A, 321, 268

Smartt, S. J., Crowther, P. A., Dufton, P. L., et al. 2001, MNRAS, 325, 257

Smith, L. J., Norris, R. P. F., \& Crowther, P. A. 2002, MNRAS, 337, 1309

Soberman, G. E., Phinney, E. S., \& van den Heuvel, E. P. J. 1997, A\&A, 327, 620

Terlevich, R., Melnick, J., Masegosa, J., Moles, J., \& Copetti, M. V. F. 1991, A\&AS, 91, 285

Underhill, A. B., Greve, G. R., \& Louth, H. 1990, PASP, 102, 749

Van Bever, J., Belkus, H., Vanbeveren, D., \& Van Rensbergen, W. 1999, New Astronomy, 4, 173

Van Bever, J., \& Vanbeveren, D. 1998, A\&A, 317, 487

Van Bever, J., \& Vanbeveren, D. 2000, A\&A, 358, 462

Vanbeveren, D., \& Conti, P. S. 1980, A\&A, 88, 230
Vanbeveren, D., De Donder, E., Van Bever, J., Van Rensbergen, W., \& De Loore, C. 1998c, New Astronomy, 3, 443

Vanbeveren, D., De Greve, J. P., Van Dessel, E. L., \& de Loore, C. 1979, A\&A, 73, 219

Vanbeveren, D., \& De Loore, C. 1994, A\&A, 290, 129

Vanbeveren, D., \& Packet, W. 1979, A\&A, 80, 242

Vanbeveren, D., Van Rensbergen, W., \& De Loore, C. 1998a, A\&ARv, 9,63

Vanbeveren, D., Van Rensbergen, W., \& De Loore, C. 1998b, in The Brightest Binaries (Dordrecht: Kluwer Academic Publishers)

Vanbeveren, D. 1982, A\&A, 115, 65

Vanbeveren, D. 1991, A\&A, 252, 159

Vanbeveren, D. 2001, in The Influence of Binaries on Stellar Population Studies, ed. D. Vanbeveren (Dordrecht: Kluwer Academic Publishers), 249

Van Rensbergen, W. 2001, in The Influence of Binaries on Stellar Population Studies, ed. D. Vanbeveren (Dordrecht: Kluwer Academic Publishers), 21

Vereshchagin, S., Kraicheva, Z., Popova, E., Tutukov, A. V., \& Yungelson, L. 1987, Pis ma Astronomicheskii Zhurnal, 13, 63

Vereshchagin, S., Tutukov, A. V., Yungelson, L., Kraicheva, Z., \& Popova, E. 1988, Ap\&SS, 144, 245

Webbink, R. F. 1984, ApJ, 277, 355 